\title{
Historical and Sociological Origins of Sustainability as a Cultural Process
}

\author{
Gianluca Senatore \\ Dipartimento di Comunicazione e Ricerca Sociale \\ Sapienza University of Rome \\ Email: gianluca.senatore@uniroma1.it
}

Doi:10.5901/mjss.2014.v5n4p681

\begin{abstract}
This paper aims to highlight the close connection existing between the main leading scholars, those considered the contemporary theorists of environmentalism and ecologism and some groups and movements that developed in Russia in the early twenties. The environmental crisis and its consequences, strongly perceived during the sixties and seventies, have given a strong impetus to sociology, they definitely introduced environmental issues as well as a new case study of environmental problems. Sociological disciplines use to study the relationship between man and nature. This kind of disciplines occurred at the end of the seventies of the last century. Before that time, environmental issues were not underestimated, they, rather, took scientist's interest. I would demonstrate that during the early twenties, Russia offers scientific studies, theories and relationships that in many cases inspired the majority of Western scholars seen as the leading scholars of an "environmental issue". Moreover, I would demonstrate that there was already a strong perception of the modern meaning of terms such as ecology, nature, environment, crisis, limit risk. Those concepts were used mostly to assume a social and economic development of rational type (sustainable). Clearly many authors, not only sociologists and economists, such as Ludwig von Bertalanffy, Kenneth Boulding, Nicholas Georgescu-Roegen, Riley Dunlap, William Catton and many others, have been influenced by some intellectuals, scientists and environmentalists Russians. The question is not about following a Marxistideological path, because it is evident that there is no connection with Marxism stricto sensu. The environmental issue in Russia in fact was strongly put forward in ancient times yet by the scientific community (even the anti-Marxist ones) and continued by some motions trough 1917-1929, at first well seen by the regime. Lenin and Bogdanov where the main protectors of this kind of line. Bogdanov, especially, is a leading figure that contributed with his tektology theory, with his science fiction stories loaded, with deep scientific references, with the role of man in nature, with a fear for the increase in population, with the recognition of the limits of the resources and of the same nature, 'industrial pollution and more.., he gave a real impetus to the process of cultural sustainability. This process in favor of a natural environment as well as of a sustainable development, is the same process known today. Bogdanov is simply the beginning of a tendance.
\end{abstract}

Keywords: sustainability, sustainable development, environmental sociology, limit, risk, environmental issues, environmental crisis, cultural processes.

\section{Introduzione}

La questione ambientale è stata scarsamente trattata in Russia come in Europa occidentale fino agli anni Sessanta del novecento. A partire dal 1988 Douglas Weiner, attraverso una serie di ricerche pubblicate proprio in quegli anni, ha fornito una nuova interpretazione (Weiner 2005) di quelle che sono state le scelte di politica ambientale legate allo sviluppo della Russia bolscevica prima e dell'Unione Sovietica dopo. Questa ampia e dettagliata ricerca si è basata soprattutto su una documentazione archivistica, su corrispondenza istituzionale e privata, su dichiarazioni e conclusioni scientifiche che hanno fatto emergere l'importanza delle iniziative intraprese a favore della conservazione della natura intesa come tutela e utilizzo razionale delle risorse - e dal ruolo strategico giocato dal movimento ambientalista russo (movimento per la protezione della natura russo), d'dapprima formato da un élite di scienziati e poi sviluppatosi negli anni come un vero e proprio movimento di opinione.

La ricerca di Weiner, testimonia l'esistenza di questo movimento autonomo a favore della protezione ambientale nato nel periodo leniniano e perdurato per tutta la fase staliniana e oltre. Esso si è esplicato attraverso una serie di società controllate da botanici, zoologi, e geografi in particolare: VOOP (società russa per la protezione della natura); MOIP (società dei naturalisti di Mosca); MGO (filiale moscovita della società geografica dell'URSS) e Società Botanica Unita (Weiner 1988). Queste società hanno tutte avuto il merito di fornire una visione alternativa dell'utilizzo della terra. Inoltre hanno rappresentato una visione alternativa circa lo sfruttamento delle risorse, della protezione dell'habitat naturale, dello sviluppo, arrivando a considerare come possibile e fattibile uno sviluppo economico razionale e forse, 
come vedremo, anche "sostenibile".

Tutte queste società ambientaliste erano di fatto, soprattutto dal 1929 in poi, in netto contrasto rispetto alle pratiche dei Soviet. Erano orgogliose delle proprie tradizioni (alternative); contestavano le pratiche del regime attraverso elezioni segrete e attraverso una rete di contatti e legami con scienziati stranieri (Weiner 1999). Gli elementi che emergono da questa straordinaria ricostruzione sono essenzialmente due. II primo è che questo movimento ha realmente contribuito alla pianificazione economica e sociale della Russia tra il 1918 e il 1924. Il secondo elemento è particolarmente significativo, poiché ne conferma il primo - il ruolo primario che il movimento per la conservazione della natura ha avuto con Lenin - e descrive uno scenario sociale e culturale carico di fermenti intellettuali, posizioni critiche, autonomie e autenticità di approccio dell'espressione di nuove idee, che si manifesteranno in modo profondo con l'arrivo di Stalin fino ad acutizzarsi fortemente nel secondo dopoguerra. Ma questo elemento di contraddizione e critica dei governi e del loro operato, da parte del movimento, nascerà solo nel momento in cui i soviet non considereranno più la tutela della natura come scelta prioritaria dell'agenda di governo. Occorre specificare che il movimento per la protezione della natura e tutte le società di riferimento, avevano incominciato le proprie attività - non ancora in modo organico prima della rivoluzione bolscevica. Gli scienziati e gli accademici, iniziatori di questo movimento, avevano accolto con piacere la cacciata dello Zar, visto dagli stessi un ostacolo alla realizzazione dei progetti per la protezione della natura. Dunque la rivoluzione bolscevica veniva considerata - per via della sua ispirazione anche positivistica - come una grande opportunità per la costruzione di una società ispirata ad uno sviluppo integrale che comprendesse uno sviluppo sociale ed economico fortemente controllato, con alcune eccezioni. Dunque, quale migliore occasione di quella rivoluzionaria per la costruzione di una società socialista che comprendesse nelle sue priorità la tutela della natura?

È evidente che questa particolare vicenda, per nulla dibattuta e tantomeno descritta fino ad oggi, ci obbliga a fare un'analisi ad ampio spettro partendo dal rapporto tra marxismo ed ecologismo.

\section{Marxismo, ambientalismo e prime teorie sull'economia ecologica.}

Arran Gare, Professore di filosofia ambientale presso la Facoltà di scienze sociali della Swinburne University of Tecnology, ci offre un interessante spunto per costruire alcuni passaggi utili a semplificare il nostro percorso e a consolidare la nostra teoria.

Secondo Gare, risulta sorprendente che i marxisti dell'Unione Sovietica affermassero che la ragione ultima per sostituire il capitalismo con il socialismo risiedesse nella crisi ambientale. A questo proposito cita le parole Arkadii Dmitrievich Ursul: «La crisi dell'ambiente che si sta sviluppando ovunque, coincide con l'ultimo stadio della crisi generale del capitalismo. La convinzione che si sta diffondendo è che solo il collasso del sistema capitalistico e la vittoria del socialismo nel mondo possa creare una fondamentale e generale opportunità per un utilizzo razionale delle risorse e un alto livello di interazione ottimale con la natura. Tale verosimile evidenza risiede nel fatto che il socialismo è una condizione necessaria per ottimizzare le relazioni tra la società e la natura, e che esso così com'è agisce nel segno di una politica rispettosa dell'ambiente» (Ursul 1983).

Una delle sfortunate eredità del comunismo sovietico è stata quella di aver lasciato nella più totale oscurità molto del passato russo (Gare 1996). Questo complesso rapporto tra passato e presente nasconde ancora molte informazioni sull'articolata organizzazione - intesa come struttura sociale, economica e politica - dell'Unione sovietica e sui processi sociali e culturali - sorprendentemente ignorati - che solo oggi, attraverso studi specifici, stanno riemergendo con non poche difficoltà. Una tra tutte, è la straordinaria riscoperta di un complesso processo culturale che ha gemmato, in un periodo lontano da ogni sospetto e in una fase storica particolarmente complessa, il primo movimento ambientalista in grado di determinare scelte culturali ma anche di politica economica.

Dal rovesciamento del comunismo erano nati movimenti ambientalisti molto variegati, antimarxisti, nazionalisti, radicali e anarchici (Pryde 1972; 1991; De Bardeleben 1985). Essi non erano consapevoli del fatto che un movimento ambientalista molto forte era nato dalle stesse radici del bolscevismo (Komarov 1978). Tale ambientalismo, nelle sue radici bolsceviche aveva tentato una sintesi di socialismo e anarcosindacalismo ed era stato assimilato dall'Occidente con i marxisti dell'ovest, contrari sia al controllo della società da parte dei mercati sia alla dominazione della società da parte di burocrazie statali centralizzate (Gare 1996).

Le origini dell'ambientalismo in Russia portano indietro nel tempo, anche prima della rivoluzione vi erano una serie di movimenti per l'ambientalismo bolscevico. Nella storia dell'ambientalismo russo che copre il periodo fino al 1935 in modo profondo, Douglas Weiner fa notare l'impegno posto in essere da parte di Lenin.

Lenin aveva una fiducia estrema nei confronti della scienza - altro elemento caratterizzante di questo periodo - e si impegnò tanto nella creazione di una società organizzata per lo sviluppo di tutte le scienze, quanto per una politica 
mirata alla tutela ambientale. L'ambientalismo di Lenin sebbene di rilievo e per così dire illuminato, secondo Gare, non venne mai interpretato come autentica visione di uno sviluppo razionale (Gare 1996; Pannekoek 1932). A mio parere tanto Bogdanov quanto Lenin, in forme diverse e con responsabilità diverse, contribuirono a favorire una percezione della natura e dell'ambiente come elemento - non solo fisico-biologico - fondamentale per lo sviluppo di una società moderna. Possiamo riscontrare in Lenin, proprio in una delle sue pubblicazioni dal titolo Materialismo ed empiriocriticismo, scritta in polemica con lo stesso Bogdanov, la convinzione che le leggi di natura dovessero esser poste come fondamento e limite all'attività umana: "Le leggi del mondo esterno, della natura... sono il fondamento dell'attività finalistica umana" (Lenin 1973, 352-54).

II marxismo così come era concepito da Struve, Plekhanov e Lenin, forniva una ideologia che permetteva di appropriarsi della campagna occidentale a favore dello sviluppo tecnologico e contemporaneamente di continuare una campagna contro le società capitalistiche (Gare 1996).

Ė impossibile comprendere la politica dell'Unione Sovietica, orientata verso l'oppressione e la tecnologizzazione e contemporaneamente verso questa lotta contro l'Occidente, se non in relazione alle minacce continue che provenivano da Ovest. In ogni caso, molte idee anche più radicali di quelle di Lenin furono non solo promosse ma anche poste in essere. II tema centrale era quello di creare una società socialista che sarebbe poi servita alla costituzione di una nuova cultura che tra l'altro avrebbe trasformato la relazione tra il genere umano e l'ambiente. Lo stesso antropocentrismo marxiano e il rapporto uomo natura, può essere interpretato come comprensione della natura da parte dell'uomo e non dominio su di essa (Dickens 1997).

Nel settembre del 1918, il Proletkul't (Organizzazione Proletaria per la Cultura e l'Educazione) un'istituzione artistica sovietica sperimentale nata con la rivoluzione, ebbe la sua prima conferenza ufficiale. Questo contenitore culturale veniva considerato di fondamentale importanza per la sinistra bolscevica, che all'indomani della fallita rivoluzione 1905-1907 si collocò all'opposizione del partito bolscevico dichiarando posizioni molto radicali. II leader dei bolscevichi radicali era Aleksander Bogdanov, storico avversario di Lenin nel partito ma anche amico nella prima fase della loro conoscenza. Per comprendere le idee di Bogdanov e il loro significato è necessario considerare il suo lavoro in relazione alla visione politica e la concezione prospettica del suo gruppo di riferimento, i cosiddetti idealisti.

Questi pensatori erano stati influenzati dall'energetica e dalla termodinamica. II loro empirismo era stato elaborato come parte degli sforzi per superare il dualismo tra materia e ragione legata a una visione meccanicistica del mondo. Tale filosofia vista in relazione alla storia del positivismo logico, fu notevolmente ignorata e spesso mal interpretata. Juan Martinez Alier, considera questo gruppo di pensatori, tutti socialisti anche se con peculiarità diverse, come i fondatori dell'economia ecologica. Il primo a sviluppare una serie di teorie su questa scia fu Sergei Podolinsky, naturalmente influenzato dalla teoria economica di Marx e da Engels. Egli cercò di riformulare la teoria di Marx sul plusvalore in termini fisici come appropriazione dell'energia utilizzabile, con ciò focalizzandosi sui limiti dell'ambiente naturale, sul modo in cui i contadini venivano sfruttati dagli altri. Tali idee vennero poi sviluppate indipendentemente da Edward Sacher, Leopold Pfaundler, Josef Popper Lynkeus, Wilhelm Ostwald, Ernst Mach, Fredrick Soddy e Otto Neurath.

\section{Bogdanov e la Tectologia, il primo ispiratore delle teorie moderne sulla sostenibilità.}

Aleksandr Bogdanov era uno studente di medicina, era un Narodnik e un membro del Narodnaja Volja ovverosia la Volontà del Popolo, gruppo che assassinò Alessandro II nel 1881 (White 1981). Influenzato in particolare da Georges Sorel, egli fu il fautore della scissione con Lenin sull'organizzazione sindacale. Egli anelava un collettivo vivente, un sindacato come base di tutti gli sforzi proletari. Su questo, nel 1908, si consumò la rottura dei bolscevichi.

Ci furono due fasi intellettuali nella carriera di Bogdanov. La prima era rivolta al superamento del conflitto con la borghesia, passando attraverso una nuova cultura che si alimentasse dello strumento della scienza. È molto probabile, come sostiene lo stesso Gare, che alcune idee di Gramsci derivassero da una riflessione su Bogdanov (Sochor 1981; Gare 1996). Anticipando le critiche marxiste da parte della scienza al rapporto tra capitalismo e marxismo, egli interpretò l'elemento meccanico del mondo come lo spartiacque tra materia e ragione, idealismo e materialismo, espressioni delle pratiche sociali della società capitalistica del feticismo delle comodità, implicazioni delle relazioni di mercato, le quali in definitiva scindevano l'aspetto organizzativo e quello esecutivo del processo di lavoro (Gare 2000a). Bogdanov spingeva per un rigenerazione culturale basata sui metodi di comprensione della società, metodi che prevedessero un superamento delle divisioni tra lavoro manuale e mentale e lavoro organizzativo ed esecutivo.

La seconda fase intellettuale di Bogdanov lo consacrò alla scienza come colui che aveva fornito gli strumenti per realizzare tale modello. II suo lavoro più importante, che ispirò molti intellettuali e scienziati, aveva come oggetto la Tectologia, una scienza universale e di tipo organizzativo (Bogdanov 1980). In tale opera pubblicata tra il 1913 e il 1922, 
egli sviluppava le idee degli energeticisti come teoria generale dell'organizzazione. Questa nuova scienza proletaria preconizzava la teoria dei sistemi del biologo Ludwig von Bertalanffy e dell'economista Kenneth Boulding, che a partire dal 1954 - anno in cui si incontrarono scienziati europei e americani a Palo Alto - avrà un ruolo fondamentale nel isomorfizzare tra loro settori conoscitivi tradizionalmente separati (Boulding 1966, 3-14). II concetto di sistema offrirà infatti la possibilità di porre in relazione tra loro ambiti tradizionalmente studiati secondo modalità esclusivamente specialistiche e spingerà molti studiosi ad ipotizzare applicazioni di questa teoria alla questione ambientale. Uno tra i più importanti autori che possiamo ricordare è Nicholas Georgescu-Roegen, che nel 1971 descriverà come l'entropia dell'universo fisico aumenta costantemente dato il continuo degrado qualitativo e irreversibile dell'ordine del caos. "La natura entropica del processo economico, che erode le risorse naturali e altera l'ambiente, costituisce un grave pericolo. L'uomo deve imparare a razionalizzare le poche risorse disponibili se ha l'intenzione di pianificare la sua esistenza nel lungo periodo» (Georgescu-Roegen 1971).

Dunque, la Tectologia avrebbe dovuto fornire una visione armonica e unitaria tra l'aspetto spirituale, culturale, e fisico dell'esperienza del collettivo di lavoro. In essa, scienza e attività dovevano essere organizzate e tutto il passato doveva essere ripensato in funzione di ciò (Gare 2000a). Linguaggio e carattere comune degli elementi erano principi dell'organizzazione, cosa che li connetteva l'un l'altro (Gare 2000b). Dalla concezione dell'uomo come parte della natura, come essere esistente solo in termini di ottenimento e utilizzo dell'energia, Bogdanov giunse a porre i limiti dell'ambiente sotto la lente d'ingrandimento (Bogdanov 1984, 116-117). In un altro fondamentale lavoro a carattere apparentemente divulgativo e propagandistico, basato sulla metafora del pianeta Marte, Bogdanov immagina la storia di un comunista rivoluzionario che dopo la rivoluzione viene portato su Marte e che in un primo momento vede gli effetti della rivoluzione come armoniosi, poi comprende come l'armonia sia superficiale e nasconda gli effetti di un successo forzato. Le industrie sono inquinanti, molte sono state quindi trasferite sotto terra. La popolazione cresce con una tale rapidità che la scarsità e la miseria sono radicate. Le risorse sono finite, le foreste sono distrutte e si dibatte su come trovarne al di fuori (Bogdanov 1984). Di fatto sono anticipati tutti i temi che saranno sviscerati dai movimenti ambientalisti, da tutti gli scienziati e i teorici della sostenibilità. Non possiamo non evidenziare come tali teorie siano in completa armonia con alcuni principi ispiratori della sociologia ambientale. II nuovo paradigma ecologico di Riley Dunlap e William Catton e i suoi principi caratterizzanti erano stati già descritti da Bogdanov: l'uomo fa parte della comunità biotica e ne è una tra le tante specie che la abita; le azioni dell'uomo producono conseguenze inattese innescando meccanismi di retroazione; la terra è limitata e non può supportare una crescita illimitata delle attività dell'uomo; anche se l'uomo ha la capacità di superare i limiti di carico dell'ambiente, lo stesso deve avere la volontà di riconoscere quei vincoli definiti dall'ambiente biologico (Dunlap e Catton 1978). Questa particolare affinità tra le teorie di Bogdanov e i principi del NEP di Dunlap e Catton ci fanno suppore che il due studiosi americani siano stati influenzati da questo particolare filone di studi scientifici. Probabilmente il condizionamento non sarà stato così diretto come noi oggi supponiamo, ma è evidente che queste teorie hanno una collocazione storico-sociologica e un'origine filosofico-politica ben definita. Dunque possiamo affermare che in Bogdanov ritroviamo in qualche modo non solo quella che sarà la critica al marxismo, ma soprattutto l'ispiratore delle principali teorie sul concetto di limite, di entropia e di rischio legato all'ambiente.

\section{Da Bogdanov a Lenin}

È importante comprendere a questo proposito come si sviluppa il movimento Proletkul't e quale peso ebbe su tutta la questione culturale ed ambientale che da qui in poi diverrà non solo un lungo processo culturale ma anche un forte elemento di influenza nelle politiche di Lenin. Tale movimento fu ispirato e diretto da Bogdanov (Malley 1990; Gare 1996). Questo movimento contò nel suo momento migliore 400,000 membri e fu l'attrattiva di artisti, musicisti, scrittori, nonché ispiratore e riferimento per moltissime riviste e quotidiani. Nel 1919, Bogdavnov costituì anche l'università proletaria di Mosca. Tutto questo fervore, ebbe una eco in Occidente soprattutto nei filo marxisti e fu oggetto di attacco da parte di Lenin che vedeva tutto questo come una minaccia sindacalista alla propria filosofia politica e alle istituzioni che stava costituendo. Lenin che concepiva la storia in termini dialettici come conflitto tra una spontanea e consapevole direzione, una storia in cui il progresso si ottiene attraverso il controllo della spontaneità attraverso la ragione, condannava tali tendenze sindacali come disordine infantile (Lenin 1975; Gare 1996).

Nel 1920, dopo diversi e ripetuti contrasti, Lenin fece subordinare il Prolekul't al Commissariato per l'Educazione e successivamente abolire. Nonostante tutto, la figura di Bogdanov continuava ad esercitare un forte fascino nei confronti di buona parte di scienziati ed intellettuali e naturalmente non poteva essere diversamente nei confronti del suo grande amico Anatolij Vasil'evic Lunacharskij. Quest'ultimo, divenne commissario per l'Educazione nel 1917 e vi rimase fino al 1929, quando rassegnò le dimissioni. Lunacharskij fu una delle figure più importante di quegli anni. Lenin riponeva in 
Lunacharskij una fiducia ed una stima incondizionata e il fatto di essere a sua volta amico ed estimatore di Bogdanov non influì minimamente sul loro rapporto, anzi.

II periodo tra il 1917 e il 1929 fu considerato un periodo d'Oro per la cultura sovietica. Tra i successi del Commissariato presieduto da Lunacarskij, l'incremento di attività culturali, di politiche pluralistiche, di dibattito scientifico e soprattutto di forte considerazione del ruolo della natura come espressione principale di uno sviluppo armonico, che si concretizzò con l'istituzione di parchi e di riserve naturali totalmente inviolate da ogni tipo di sfruttamento industriale 0 agricolo.

Con il commissariato di Lunacharskij si era avuta l'idea di come dovesse essere una reale scienza socialista. Per Alexander Vucinich gli sviluppi di questa fase storico-culturale rappresenta un revival del nichilismo russo. Lo stesso Vucinich nello studio sull'Accademia delle Scienze dell'Unione Sovietica (Vucinich 1984), concepisce gli scienziati degli anni venti del secolo come fautori della ricostituzione della scienza dopo il caos della Grande Guerra e della guerra civile. È difficile interpretare questo periodo. Di certo possiamo affermare che in questa fase l'indipendenza degli scienziati non fu certo messa in discussione.

La fase sovietica leniniana, senza alcun dubbio, ha determinato le condizioni di siffatto processo culturale. È difficile, ad oggi, immaginare o ipotizzare scenari differenti da quelli ai quali ci rivolgiamo. Quasi tutto quello che oggi ritroviamo nella costruzione del pensiero ambientalista-ecologista, base fondamentale per l'individuazione dei principi ispiratori del concetto di sostenibilità, come abbiamo avuto modo di verificare, è stato profondamente stimolato da questi processi sociologico-culturali sviluppatisi nella Russia leniniana.

Negli anni venti, la questione ambientale era uno dei temi più dibattuti in Russia. Basti pensare che uno degli scienziati più conosciuti in Europa e nel Mondo, Vladimir Ivanovič Vernadskij, fu invitato in Francia nel 1923 a discutere dei problemi legati al degrado antropico. Vernadskij e altri importanti scienziati e filosofi come Marie-Joseph Pierre Teilhard de Chardin o Edouard Le Roy, già nel 1923, iniziarono il dibattito sulla Noosfera (la sfera della ragione con capacità intellettuale e tecnologica di plasmare la Terra o meglio la biosfera) ipotizzando che la stessa potesse essere considerata un involucro geografico della sfera terrestre, nel quale si accumulano tutte le trasformazione della materia, dell'energie e le informazioni collegate all'attività dell'uomo dotato di ragione (Vernadskij 1993). Questo sviluppo della scienza che andava incontro ad una nuova relazione tra l'ambiente naturale e la società era senza dubbio legato alla presenza del movimento per la protezione della natura (Weiner 1988).

Già prima della rivoluzione si era registrata la presenza di ambientalisti sul territorio russo. Naturalmente questi, non ancora organizzati nella forma e nella struttura che ritroveremo dopo il 1917, provenivano sempre dal mondo scientifico e furono comunque gli iniziatori del movimento per la protezione della natura. II contributo, non indifferente alla comunità scientifica, fu completamente ignorato dall'Europa e dagli Stati Uniti (Weiner 1988), e solo dopo molti anni alcuni di questi scienziati ebbero il giusto riconoscimento. Innanzitutto ci furono coloro che erano impegnati sulla valutazione economica dei danni ambientali dovuti all'utilizzo delle risorse naturali. Secondariamente c'erano coloro che volevano recuperare il significato autentico della natura e volevano riconoscere il diritto ad un'esistenza armoniosa e ad un buon livello di salute fisica come Aleksandr Porfir'evič Borodin (Gare 1996). Un terzo gruppo, molto interessante dal nostro punto di vista, si occupava di studiare e sviluppare l'ambito della fitosociologia, lo studio di comunità vegetali. Questi pionieri guardavano alla natura come modello di armonia, efficienza, produttività che gli agricoltori avrebbero dovuto emulare. In sostanza, secondo questo terzo gruppo, la natura doveva essere studiata nella sua forma autentica di purezza, senza nessun condizionamento esterno. Attraverso questi studi sarebbe stato possibile applicare ad altri sistemi complessi i risultati scientifici. Fu attraverso queste richieste che vennero istituite vaste aree protette, dette zapovedniki (parco naturale).

L'idea di istituire le zapovedniki - riserve naturali completamente incontaminate da qualsiasi intervento dell'uomo fu proposta per la prima volta dallo scienziato russo Grigorij Aleksandrovic Kozhevnikov nel 1909. Lo scopo di questo intervento a favore della natura aveva il doppio obiettivo di tutelare la natura e utilizzare queste riserve a scopi scientifici per monitorare e studiare le specie animali e vegetali in riferimento all'evoluzione della natura senza condizionamenti esterni. Da qui nasce il movimento per la protezione della natura che darà vita ad una serie di associazioni come la VOOP, la Moscow Society of Naturalists (MOIP), la Ali-Union Botanical Society, la Moscow branch of the Geographical Society of the USSR (MGO). È significativo che il maggiore impulso legato a queste attività di salvaguardia ambientale si osserverà proprio dopo 1917.

Benché Lenin non avesse mai affrontato in modo particolarmente approfondito la questione sulla possibilità di considerare l'ambiente e la natura una risorsa da tutelare, fra i dirigenti del partito si era dimostrato da subito uno dei più attenti all'argomento. II pessimo rapporto con Bogdavnov, considerato da molti il principale ispiratore del movimento ambientalista russo, non influenzò negativamente Lenin, anzi. La gestione delle risorse e il rispetto delle leggi della 
natura accompagnavano Lenin nella sua idea di incrementare la produzione industriale nella Russia bolscevica. Da queste considerazioni è intuibile, come ci ricorda lo stesso Weiner (1999), che nei progetti di Lenin non c'era solo l'intenzione di stabilire un rapporto con il mondo accademico e con l'eredità scientifica e culturale borghese per elevare il livello tecnologico del Paese, ma si consolidava l'idea di un necessario legame tra il partito e gli scienziati che si occupavano proprio delle scienze naturali. La conferma di questo specifico progetto si evince da subito attraverso le concrete azioni politiche del governo sovietico, che nell'aprile del 1918 firma un accordo con l'Accademia delle Scienza. Lo stesso accordo, sottoscritto dal Commissario del popolo per l'istruzione Lunacarskij - amico e profondo estimatore di Bogdavnov - prevedeva il riconoscimento dell'autonomia e dell'indipendenza alle istituzioni scientifiche ed accademiche in cambio di una leale collaborazione. A distanza di un anno viene costituito il Comitato dello Stato per la Protezione dei Monumenti della Natura con la partecipazione di molti illustri scienziati e studiosi russi tra i quali: l'antropologo e geografo Dimitri Anuchin, il geochimico e mineralogista Alexander Fersman. L'11 aprile del 1919 viene istituito il primo zapovedniki (parco naturale) della Repubblica Socialista Federativa Sovietica Russa. La particolarità di questo evento è dato dal fatto che lo stesso Lenin accettò di occuparsi personalmente di questo progetto che gli era stato proposto da Nikolaj Nikolaevic Podiapolskij, un agronomo bolscevico, al quale diede l'incarico di predisporre una serie di proposte per l'istituzione di riserve e parchi su tutto il territorio sovietico (Weiner 1988). II 14 maggio 1920 nasce l'Ilmenskij zapovedniki (parco nazionale) nella regione di Miass negli Urali meridionali con decreto istitutivo firmato da Lenin. Lo stesso Lenin nel 16 settembre 1921 firma anche il decreto per "la protezione dei monumenti della natura, le riserve e i parchi" ed affida al Commissario del popolo per l'istruzione tutte le competenze in materia di salvaguardia del patrimonio naturale con vincoli strettissimi sullo sfruttamento delle aree protette. Inoltre, da questo momento in poi, il Commissario acquisirà pieni poteri per l'istituzione di nuovi zapovedniki su tutto il territorio sovietico. La scelta di affidare il compito al Commissariato per l'istruzione nasce dal fatto che questo ministero difficilmente avrebbe potuto sfruttare economicamente le zapovedniki.

Tali aree rimasero per lungo tempo inviolate, o quasi, fino al 29 agosto 1951 quando Stalin con il decreto n. 3192 "O zapovednikach" fece rimuovere i vincoli di tutela da 88 riserve naturali su $128{ }^{1}$.

Per comprendere alcune delle idee portate avanti dagli ecologisti - termine appropriato per descrivere la funzione di questi personaggi, anticipatori del moderno ecologismo - si può guardare ad una figura di rilievo come quella di Stanchinskii che divenne uno dei punti di riferimento per l'ambientalismo-ecologismo dell'Unione Sovietica. Egli dimostrò come le biocenosis (ecosistemi o comunità biologiche) fossero caratterizzate da una stabilità relativa, ovverosia un equilibrio dinamico nel quale un determinato numero di elementi diversi rimane costante nel tempo a discapito della capacità di proliferare in modo esponenziale. Stanchinskii costruì un modello matematico che descriveva l'energia prodotta annualmente da una biocenosis, riuscì a misurare la biomassa dei componenti e in definitiva costituì il primo studio applicabile su modelli di questo genere (Gare 1996).

Ciò che rese la figura di Stanchinskii emblematica per questo periodo storico non furono solo le sue idee ma soprattutto il fatto che poté svolgere i suoi studi con un forte appoggio da parte del regime. Le scoperte e il fermento di quegli anni furono possibili solo grazie al Commissariato per l'educazione. Ė giusto ricordare, inoltre, che Stanchinskii fu ispirato da Vernandskij, allora membro dell'Accademia delle Scienze. Vernandskij a sua volta era in totale accordo con le teoria di Bogdanov, della Tectologia e del Proletkul't.

Era evidente che le scoperte di Stanchinskii avvaloravano la tesi secondo la quale il ruolo della scienza non dava solo lustro ai singoli scienziati ma a tutta la Russia. Stanchinskii non aveva solo scoperto la capacità delle masse di produrre energia, ma aveva dato conferma che lo sviluppo di un Paese passa attraverso l'innovazione e la ricerca scientifica. I fondi per queste ed altre ricerche aumentarono e l'armonia con il Partito e il regime non stentò ad arrivare, anche se non durò a lungo.

Stanchinskii ebbe un ruolo fondamentale anche nel Congresso del 1929 quando affermò che gli scienziati avrebbero dovuto contribuire in modo determinante nel Piano Quinquennale, avrebbero dovuto rivedere e monitorare l'andamento dei piani di sviluppo rendendoli possibilmente più armoniosi con le risorse a disposizione (Gare 1996).

È fuori dubbio che le azioni politiche del governo Russo dal 1918 al 1924 furono fortemente influenzate dall'élite accademica e scientifica del movimento per la protezione della natura russo. Questa riuscì a incidere anche sulla politica di sviluppo economico almeno fino alla morte di Lenin. Infatti, già prima dell'introduzione del primo Piano Quinquennale

${ }^{1}$ Con il decreto O zapovednikach voluto da Stalin il territorio dei zapovedniki passarono da 12,6 milioni di ettari a circa 1,384 milioni di ettari. Lo sfruttamento delle riserve e dei parchi tutelati fino ad allora dalla legge "sulla protezione dei monumenti della natura, le riserve e i parchi" del 16 settembre 1921, non coinvolse solo la Russia ma anche tutte le altre repubbliche che dovettero promulgare decreti simili per consentire lo sfruttamento delle aree naturalistiche protette. L'Ucraina, la Georgia e la Lituania ne persero rispettivamente 19, 16 e 13 e le altre repubbliche circa 10 ciascuna (Weiner 1999, 129; Dundovich 2012, 45). 
che avrebbe sostituito la NEP avviata da Lenin nel 1921, il movimento aveva perso la sua autorevolezza soprattutto sul piano economico razionale e cioè sul fatto di favorire uno sviluppo attento allo sfruttamento delle risorse. Molti scienziati provarono a ostacolare apertamente i piani economici di Stalin ma lo sforzo fu del tutto vano.

Con l'intervento di Stalin nella sfera culturale e scientifica tutte le iniziative vennero messe in discussione. Di fatto gli ecologisti fallirono nell'intento di incidere sul Piano Quinquennale, ma divennero i principali critici di ogni implementazione del piano considerandone le ripercussioni ambientali e le possibili conseguenze di uno sfruttamento intenso delle risorse (Gare 1996).

L'esperienza di Bogdanov, di Lunacharsky e anche di questo Lenin, poco conosciuto e per nulla studiato, deve essere oggi riconosciuta e rivalutata sotto nuovi aspetti. L'influenza che questo periodo storico ha avuto nei confronti di molti accademici e studiosi degli anni sessanta e settanta nel secolo scorso, non può e non deve essere considerata solo attraverso la strada ideologica. Sarebbe un grave errore epistemologico se ancora oggi non considerassimo questa straordinaria esperienza come la prima iniziativa politica, economica e sociale che ha fortemente condizionato la società russa e quella di gran parte dell'Europa e degli USA sulla questione ambientale. Uno studio più approfondito con un approccio meno specialistico di quello utilizzato fino ad oggi, ci aiuterebbe a comprendere quei fenomeni che stiamo cercando di indagare. Dunque, non è un azzardo affermare che gran parte dei caratteri e degli elementi che stiamo oggi studiando e rapportando al processo di sostenibilità (ambientale, sociale, economica...) possono essere ricondotti nella loro genesi a questo particolare spazio temporale.

\section{Reference}

Bogdanov, Aleksandr. 1980. Essays in Tektology: The General Science of Organization. Trad. by George Gorelik. Seaside California: Intersystems Publications. (Tektologiya: Vseobschaya Organizatisionnaya Nauka, in 3 vol. Berlin and Petrograd-Moscow, 1922)

Bogdanov, Aleksandr. 1984. Red Star: The first Bolshevik Utopia. Ed., Loren R. Graham and Richard Stites. Bloomington: Indiana University Press.

Boulding, Kenneth E. 1966. "The economics of the coming Spaceship Earth." In Jarrett H., edited by, Environmental Quality in a Growing Economy. Baltimore: Johns Hopkins University Press.

Catton, R. William Jr., and Reley E. Dunlap. 1978. "Paradigms, Theories, and the Primary of the HEP-NEP Distinction." The American sociologist 13.4: 252-256. doi: 10.2307/27702344

De Bardeleben, Joan. 1985. The Environment and Marxism-Leninism: the Soviet and East German Experience. Boulder-Colo: Westview Press.

Dickens, Peter. 1990. Urban Sociology. Society, Locality and Human Nature. Hemel Hempstead: Harvester Wheatsheaf.

Dundovich, Elena. 2012. Cornobyl'. L'assenza. Firenze: Passigli Editore.

Fitzpatrick, Sheila. 1970. The Commissariat of Enlightenment. Soviet Organization of Education and the Arts under Lunacharsky, 19171921. Oxford University Press.

Gare, Arran. 1996. "Soviet Environmentalism: The Path Not Taken." in Benton T., edited by, The Greening of Marxism, New YorkLondon: The Guilford Press.

Gare, Arran. 2000a. "Aleksandr Bogdanov's History, Sociology and Philosophy of Science" Studies in History and Philosophy of Science Part A. 31.2: 231-248. doi: 10.1016/S0039-3681(00)00002-9

Gare, Arran. 2000b. "Aleksandr Bogdanov and Systems Theory" Democracy \& Nature. 6.3: 341-359. doi: 10.1080/10855660020020230

Georgescu-Roegen, Nicholas. 1971. The Entropy Law and the Economic Process, Cambridge MA: Harvard University Press.

Komarov, Boris. 1978. The Distruction of Nature in the Soviet Union. London: Pluto Press.

Lenin, Vladimir I. 1973. "Materialismo ed empiriocriticismo" in Opere scelte, III vol., Mosca/Roma: Editori Riuniti/Progres.

Lenin, Vladimir I. 1975. "Left-Wing Communism An Infantile Disorder." In Tucker Robert C., edited by, Lenin Anthology, New York: Norton.

Mally, Lynn. 1990. Culture of the Future: The Proletkult Movement in Revolutionary Russia. Berkeley: University of California Press.

Martinez Alier, J, and Naredo, J.M. 1982. "A Marxist Precursor of Energy Economics: Podolinsky" The Journal of Peasant Studies. 9. 2: 207-224. doi: 10.1080/03066158208438162

Pannekoek, Anton. 1932. Lenin as Philosopher, London, Merlin Press.

Pryde, Philip R. 1972. Conservation in the Soviet Union. Cambridge: Cambridge University Press.

Pryde Philip R. 1991. Environmental Management in the Soviet Union. Cambridge: Cambridge University Press.

Sochor, Zenovia A. 1981. "Was Bogdanov Russia's answer to Gramsci?" Studies in Soviet Thought. 22. 1: 59-81. doi: 10.1007/BF00837972

Ursul, A. Dmitrievich. 1983. Eds. Philosophy and the Ecological Problems of Civilisation, USSR Accademy of Science. Moscow: Progress Publishers

Vernadskij, V. Ivanovic. 1993. La Biosfera. Breve introduzione al concetto-chiave della moderna 'ecologia planetaria': il globo terrestre inteso come sistema vivente unitario. Como: Red.

Vucinich, Alexander. 1984. Empire of Knowledge. The Academy of Sciences of the USSR (1917-1970). Berkeley and London: 
University of California Press.

Weiner, Douglas, R. 1988. Models of Nature: Ecology, Conservation and Cultural Revolution in Soviet Russia. Bloomington: Indiana University Press.

Weiner, Douglas, R. 1999. A Little Corner of Freedom: Russian Nature Protection from Stalin to Gorbachev. Berkeley: University of California Press.

Weiner, Douglas, R. 2005. "A death-defying attempt to articulate a coherent definition of environmental history." Environmental History. 10.3: 404-420. doi:10.1093/envhis/10.3.404

White, James D. 1981. Studies in Soviet Thought. 22. 1: 33-58. doi: doi 10.1007/BF00837971 